\title{
Las Agencias de Viajes ante la influencia de las Redes Sociales en el turismo. El caso de Ourense.
}

\author{
Laura Rodríguez Cid* José Antonio Fraiz Brea** \\ David Ramos Valcárcel*** \\ Universidade de Vigo (España)
}

\begin{abstract}
Resumen: El modelo turístico tradicional ha experimentado un cambio con la llegada de las nuevas tecnologías. Las tradicionales herramientas de marketing empleadas por las Agencias de Viajes se están quedando atrás para dar paso a nuevos y mejorados métodos de comercialización y promoción de productos turísticos basados en la utilización de las Tecnologías de la Información y la Comunicación (TIC). Los hábitos de compra han cambiado debido a la rápida evolución de Internet en los últimos 10 años. Se ha pasado de la Web 1.0, de carácter estático y de lectura, a la Web 2.0 que ha generalizado la creación de contenidos y la comunicación on-line entre usuarios, convirtiéndose en creadores y a su vez en consumidores de la información. En los últimos años, las empresas turísticas han comenzado a crear canales de comercialización directos con el cliente, evitando así negociar con los intermediarios, lo que ha mejorado la eficiencia y la reducción de costes. Esto ha permitido que los usuarios puedan contratar un viaje o un servicio turístico a través de la red con unos costes notablemente inferiores. El asesoramiento que antes sólo se encontraba en las Agencias de Viajes, ahora es proporcionado por los usuarios que han consumido el servicio y cuya experiencia aporta una visión única y diferente. Todo este fenómeno ha ocasionado que este sector pase a un segundo plano, con un turista más experimentado, más exigente y familiarizado con estas nuevas herramientas nacidas de la Web 2.0. Numerosas agencias han cerrado sus negocios y tienden a desaparecer tal y como las conocemos actualmente. Sin embargo, son muchas las posibilidades que se les presentan si se adaptan al mercado actual, un entorno que fomenta el uso de las nuevas tecnologías y que utiliza la Web 2.0 y las Redes Sociales para mantener y captar nuevos clientes.
\end{abstract}

Palabras Clave: Agencias de Viajes, canales de comercialización, Web 2.0, Redes Sociales, creación de contenidos, Tecnologías de la Información y la Comunicación (TIC).

\section{Travel Agencies under the influence of social networks on tourism. The case of Ourense.}

Abstract: The traditional tourism model has changed with the appearance of new technologies. The traditional marketing tools used by Travel Agencies are disappearing, giving way to improved methods of marketing to promote tourism products which are based on the use of Information and Communication Technologies (ICT). Buying habits have changed due to the fast development of the Internet in the last 10 years. With Web 1.0, websites were static and not very interactive but now Web 2.0 allows content creation and on-line communication between users as creators and consumers of information. In recent years, tourism companies have begun to create direct marketing channels with the client to avoid dealing with intermediaries, to improve efficiency and to reduce costs. This allowed users to book a trip or a tourism service by themselves through the network and at a significantly lower price. Advice previously found in Travel Agencies is now provided by users who have used the service and whose experience brings an unique and different point of view. This whole phenomenon has forced Travel Agencies into the background because now the tourist is more experienced, more informed and familiar with these new tools born from the Web 2.0. Although interaction between the customer and the travel agent generates added value, many traditional Travel Agencies have closed their businesses and could disappear as they are known today. However, many possibilities could be opened to them if they adapt to the current market, an environment which encourages the use of new technologies from Web 2.0 to Social Networks to keep and attract new customers.

Keywords: Travel Agencies, marketing channels, Web 2.0, Social Networks, content creation, Information and Communication Technologies (ICT).

\footnotetext{
* E-mail: laurarguezcid@gmail.com

** E-mail: jafraiz@uvigo.es

*** E-mail: david@uvigo.es
} 


\section{Introducción}

Tras la llegada de la Web 2.0, las agencias on-line y los nuevos canales de comercialización, todo parece apuntar a que las Agencias de Viajes tienden a experimentar un cambio radical en su modelo tradicional de negocio; salvo aquellas que queden destinadas a atender a nichos de mercado muy concretos (Turismo sénior de IMSERSO, excursiones escolares, viajes de empresa, etc.).

Por lo tanto, el objetivo de esta investigación es analizar el panorama actual relativo a las nuevas tecnologías vinculadas al turismo, y dentro de este contexto relacionar las Agencias de Viajes de la ciudad de Ourense y sus posibilidades en el futuro a través del uso de las Redes Sociales. Esto implica evaluar cuál sería el coste y la manera de adaptarse al cambio y funcionar de forma exitosa con un nuevo modelo de negocio.

Para ello, se pretende realizar una investigación exploratoria de la presencia de las Agencias de Viajes tradicionales de la ciudad de Ourense en Internet y en las Redes Sociales constatando qué cantidad de ellas operan utilizando las nuevas tecnologías y en qué medida es necesario el cambio en aquellas que no las utilizan. Además también resulta interesante para el estudio conocer qué métodos de comercialización están utilizando y qué opinan acerca del panorama turístico actual y de su futuro como vendedores de viajes.

\section{Marco teórico}

Hasta hace poco más de una década, los métodos para comercializar productos turísticos se basaban exclusivamente en la promoción a través de las Agencias de Viajes. Contaban con los medios necesarios para vender productos y servicios turísticos al cliente que acudía a la agencia y requería ser asesorado para contratar un viaje a un destino determinado. Sin embargo, con la llegada de las Tecnologías de la Información y la Comunicación se ha creado una compleja infraestructura que ha multiplicado los canales de comunicación y distribución, que agilizan enormemente las transacciones turísticas. Además se ha generado una base de datos de usuarios que ofrecen experiencias de sus viajes a través de la red y que se nutren de éstas creando un excepcional entorno interactivo.

\section{Las Tecnologías de la Información y de la Comunicación}

Las Tecnologías de la Información y la Comunicación (TIC) han provocado una revolución sin precedentes en las formas de comunicación desde comienzos de los 90. Internet pasó de ser un instrumento usado básicamente por la comunidad científica a una red global de fácil manejo que modificó las pautas de interacción social (Vogeler y Hernández, 2000).

Las TIC se definen como un conjunto de tecnologías asociadas a las comunicaciones, la informática y los medios de comunicación, que dan soporte a la gestión de los datos de la información. Ordenadores, teléfonos móviles, tarjetas de memoria, navegación GPS (Global Position System), Internet... son algunos ejemplos de tecnologías que se han convertido en imprescindibles para las personas y que han provocado un cambio en la sociedad, que se conoce hoy en día como la "Sociedad de la información" o la "Sociedad del conocimiento" (Suárez, 2007).

Con estas nuevas aplicaciones que facilitan la difusión inmediata, el turismo se ha convertido en una actividad muy rentable. En el universo de las TIC todo ocurre a una velocidad de vértigo, evolucionando constantemente y repercutiendo de manera automática en el mercado turístico. Gracias a estos avances, las relaciones entre las agencias y el cliente han variado notablemente, de modo que los procesos de distribución y de acceso a las reservas se han vuelto mucho más dinámicos. En el caso de las agencias on-line, se han desplazado los lugares físicos que se han sustituido por espacios virtuales en los que el contacto personal se ha restringido enormemente siendo reconducido a través de la red, y que ha derivado en mejoras de costes, rapidez y eficiencia sin menoscabar la atención personalizada que se realiza. El precio se ha convertido, en la mayoría de las ocasiones, en la condición más valorada a la hora de contratar un viaje, bien sea en una Agencia tradicional o a través de Internet, y atributos como el trato personal o la calidad del servicio han quedado relegados a un segundo plano. No obstante, el cliente tiene en la red acceso a suficiente información como para hacer una valoración de la calidad de los destinos o productos turísticos de su interés.

\section{Las Redes Sociales}

Para conocer el origen de las Redes Sociales debemos remontarnos a 1967, cuando Mildgram presentaba "La teoría de un mundo pequeño" en la que fue capaz de demostrar que en 5,2 pasos de media era posible conectar a dos personas desconocidas. El experimento consistió en enviar una carta a través de una cadena de contactos a un destinatario del cual se tenía poca información. La media de pasos de las cadenas que 
lograron su objetivo fue de 5,2. Así surge la expresión que conocemos como los "seis grados de separación". Esta teoría se basaba en que vivimos en un mundo inabarcable pero a la vez muy próximo.

Cada persona en este mundo tiene una red personal. En ella se establecen una serie de lazos o uniones con otras personas con las que el individuo tiene contacto en su vida. Existen lazos más fuertes y lazos más débiles, que se crean en función de la intensidad de relación con las personas que forman parte de la red. Una aplicación de este concepto es la "hipótesis de los lazos débiles" de Granovetter (1973).

Estas reflexiones son fundamentales para poder entender desde un punto de vista más científico el funcionamiento de las Redes Sociales, pues se basan en la aplicación del fenómeno de las redes al universo virtual que llamamos Internet.

Podemos definir las Redes Sociales como una serie de servicios web que permiten a los usuarios crear un perfil público o semi-público dentro de un sistema limitado que genera una lista con otros individuos con los que se comparte alguna conexión (Boyd y Ellison, 2008). Las conexiones entre usuarios reciben un nombre determinado, que puede variar de unas Redes Sociales a otras.

Tras crear una cuenta en una Red Social, los usuarios identifican a las personas del sistema con las que tienen algún tipo de relación. Comúnmente se conoce con el término de "Amigos", "Contactos" o "Seguidores".

Lo que hace únicas a las Redes Sociales es que permiten el contacto y la comunicación entre usuarios desconocidos y la conexión entre sus redes sociales personales, es decir, las redes de contactos de cada persona a las que otros usuarios pueden acceder. De esta forma unas redes se enlazan con otras y generan un universo incalculable de redes entre personas.

Es importante hacer una pequeña reflexión acerca de los motivos que mueven a las personas a interactuar con otras y tomar parte en las Redes Sociales. Chung y Buhalis (2008) establecen tres factores por los cuales las personas utilizan las Redes Sociales:

1) Adquirir información de otros usuarios: de fácil acceso, actualizada, fiable y de confianza.

2) Sacar un provecho socio-psicológico: sentirse identificado con los otros usuarios y formar parte de una comunidad.

3) Adquirir diversión y entretenimiento.

Según estos factores y basándonos en la repercusión que tienen hoy en día las Redes Sociales en la vida cotidiana de las personas en todo el mundo, se puede decir con certeza que son una herramienta muy poderosa para ejercer influencia en millones de usuarios, atraer su atención con facilidad y generar confianza. Además su crecimiento masivo ha influido enormemente en los hábitos de comunicación, en los canales de información e incluso, en las decisiones de compra. Además su influencia en el mercado turístico es un hecho. El enorme número de usuarios que mueven ha provocado plantearse a muchos negocios y marcas estar en este mercado.

La interacción en el trato con los clientes se ha visto mejorada a través del uso de estos nuevos canales de comunicación, donde la mayoría de las cadenas hoteleras más activas, los Touroperadores e incluso las comunidades autónomas, ya han tomado fuertes posiciones. Son una potente herramienta para maximizar el uso de la comunicación y la promoción, además de una buena manera de potenciar la imagen de marca y fidelizar a los clientes aportando un feedback al sector de indudable valor añadido.

Este fenómeno ha cambiado completamente la forma de hacer marketing. No es necesaria apenas inversión para tomar presencia en las Redes Sociales, puesto que el mayor reto en el mercado actual se centra exclusivamente en la búsqueda de la reputación on-line.

Asimismo, las Redes Sociales son una herramienta cada vez más utilizada por los viajeros, en las cuales basan sus decisiones de compra a la hora de planificar viajes y elegir un destino. En ellas, es posible encontrar las opiniones sobre servicios (complejos turísticos, hoteles, restaurantes, etc.) o sobre destinos turísticos potenciales. El poder que tradicionalmente residía en los Touroperadores y en las Agencias de Viajes se ha visto desplazado en los últimos años hacia los consumidores, quienes mediante el intercambio de opiniones y recomendaciones en este tipo de redes son capaces de condicionar el comportamiento de los viajeros. Esto se debe a que a la hora de tomar una decisión de compra, los consumidores, en general, prefieren confiar en fuentes de comunicación independientes e informales (otros consumidores) en lugar de campañas publicitarias o un agente de viajes que probablemente no conozca en persona el producto o servicio que está vendiendo y cuya opinión esté sesgada por el beneficio que pretende obtener con la venta.

La reputación de una marca turística, bien sea de una empresa que preste servicios turísticos como de la imagen que se tiene de un destino turístico, establece una clara diferencia a la hora de ser elegido y recomendado por los usuarios, y además favorece la sensación de confianza, credibilidad y garantía. 
Las Agencias de Viajes, como negocios turísticos han de seguir esta línea. El hecho de que su marca sea reconocida y valorada en la red, que dé seguridad al cliente, sumado a una filosofía de venta basada en contacto directo a través de las herramientas de la Web 2.0, puede reforzar el posicionamiento de la marca en el mercado y conseguir un considerable aumento de ventas, mejorando la convivencia de la presencia on-line y off-line.

\section{Metodología}

La metodología utilizada para la elaboración de esta investigación se ha basado en la realización primeramente de un marco teórico referente a la situación actual del turismo vinculado a las nuevas tecnologías y a las herramientas de marketing turístico, junto con las Agencias de Viajes y el uso que hacen o pueden llegar a hacer de las Redes Sociales. Una vez analizado, se ha elaborado una fundamentación práctica basada en el estudio de una muestra tomada para cumplir el objeto de investigación del proyecto, cuyo fin ha sido detectar la proporción de Agencias de Viajes ourensanas que utilizan Internet y las Redes Sociales para realizar su trabajo como empresa de intermediación turística. Para ello, se ha optado por la utilización de una metodología simple consistente en la búsqueda y análisis de datos a través de fuentes de información primarias, con recogida de datos mediante cuestionario.

La investigación se inició fijando un listado de las Agencias de Viajes tradicionales emplazadas en la ciudad de Ourense que están en activo dentro del ámbito empresarial. Una vez delimitada la población objetivo en un número total de 21 establecimientos, se procedió a la elaboración de una encuesta on-line. De esta manera, se examinaron todas las unidades de población de las que se pretendía extraer los datos para el estudio. El instrumento para la recogida de los datos de cada unidad muestral ha sido un cuestionario totalmente estructurado, compuesto por un total de 14 ítems donde se preguntaba acerca de la presencia del negocio en Internet y en las Redes Sociales, el uso de las Redes Sociales, la familiarización con el concepto de la Web 2.0, los métodos de comercialización utilizados y las opiniones acerca del futuro del sector de las Agencias de Viajes tradicionales. La mayoría de las preguntas eran cerradas. El cuestionario fue enviado vía e-mail a las Agencias de Viajes seleccionadas para la muestra en el mes de Junio del año 2012 y todas ellas lo remitieron cubierto con posterioridad. Finalmente se codificaron las respuestas obtenidas para crear la base de datos y analizar los mismos.

\section{Análisis y resultados}

En cuanto a la presencia en Internet, el 80\% dispone de página web propia del negocio, por lo que en general las agencias de la ciudad están bien adaptadas a la web y al uso del correo electrónico como principal medio de comunicación. Las páginas web que más abundan son las que disponen de un buscador de viajes personalizable $(58,8 \%)$ con una serie de menús desplegables que el usuario maneja para reservar su viaje a medida. Cada día se utiliza menos la página web meramente informativa asociada al concepto de la Web 1.0. Hoy las páginas de las Agencias de Viajes combinan perfectamente información, noticias, curiosidades, su propio blog, un buscador de viajes, etc. y gestionan todas las reservas a través de Internet.

Gráfico 1: Agencias que disponen de página web propia

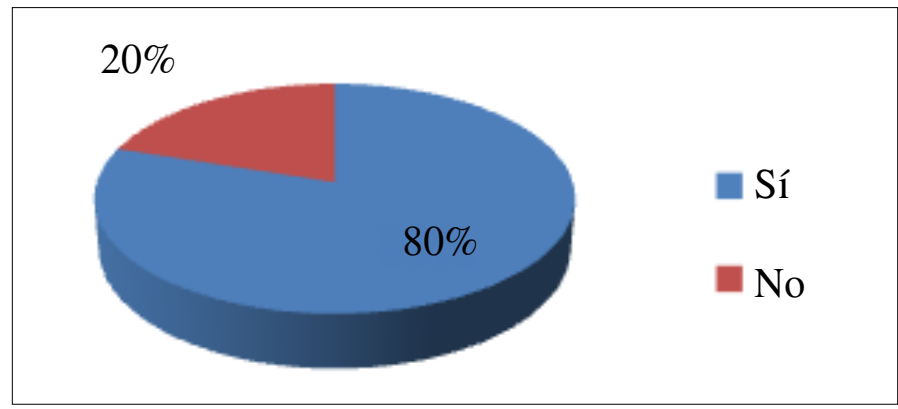

Fuente: Elaboración propia 


\section{Gráfico 2: Tipo de página web}

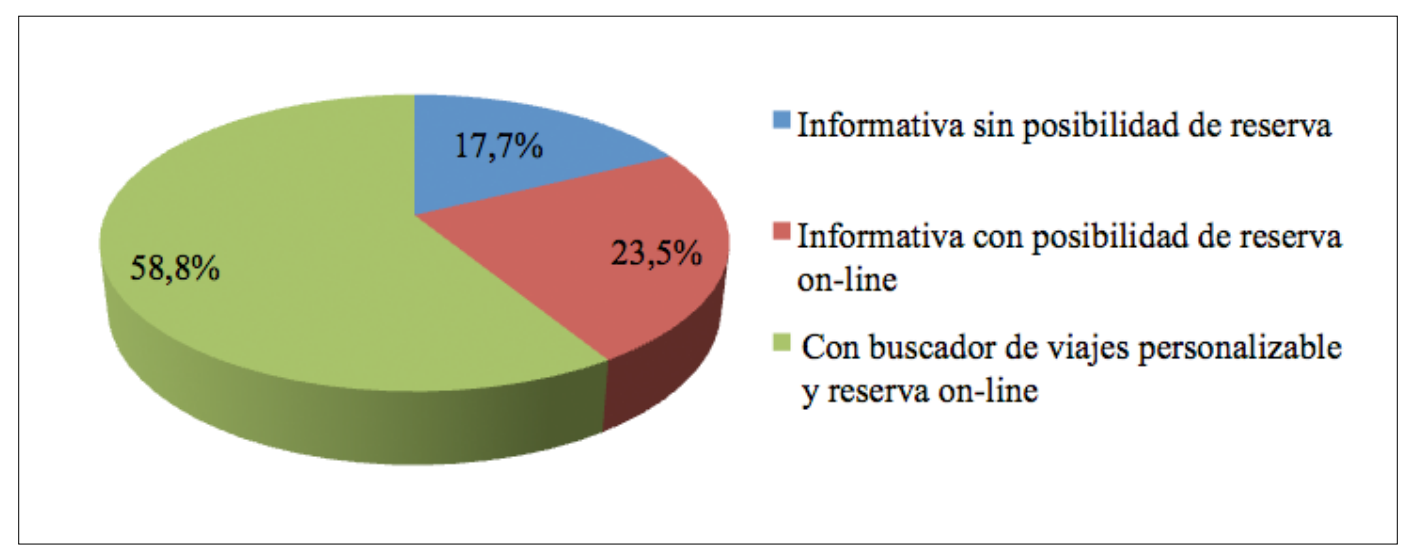

Fuente: Elaboración propia

El 86\% de los encuestados está familiarizado con el concepto de la Web 2.0 y las Redes Sociales. Sin embargo, sólo el 40\% de las Agencias emplean las Redes Sociales para su negocio. De ello se puede deducir que el uso de las Redes Sociales por parte de los agentes de viajes queda relegado exclusivamente al ámbito personal. Las que no tienen perfil en las Redes Sociales, han declarado en un 63,6\% que tienen intención de abrir cuentas a corto plazo y que saben las posibilidades que ofrecen a su negocio de cara a la interacción con los clientes. Un $27,3 \%$ ha contestado que prefieren hablar con los clientes en persona y el $9,1 \%$ restante ha manifestado que no le interesan porque creen que sólo sirven para "chatear con amigos".

En lo que se refiere a las Redes Sociales en las que tienen presencia las Agencias de Viajes, aparece Facebook con un 77,8\%, seguido de lejos por Twitter con solamente el 33,3\%. Estas agencias también tienen presencia, aunque en menor medida, en la red profesional Linkedin (22,2\%), en el canal de videos YouTube $(22,2 \%)$ y en la reciente Google+ $(22,2 \%)$.

\section{Gráfico 3: Presencia en las Redes Sociales.}
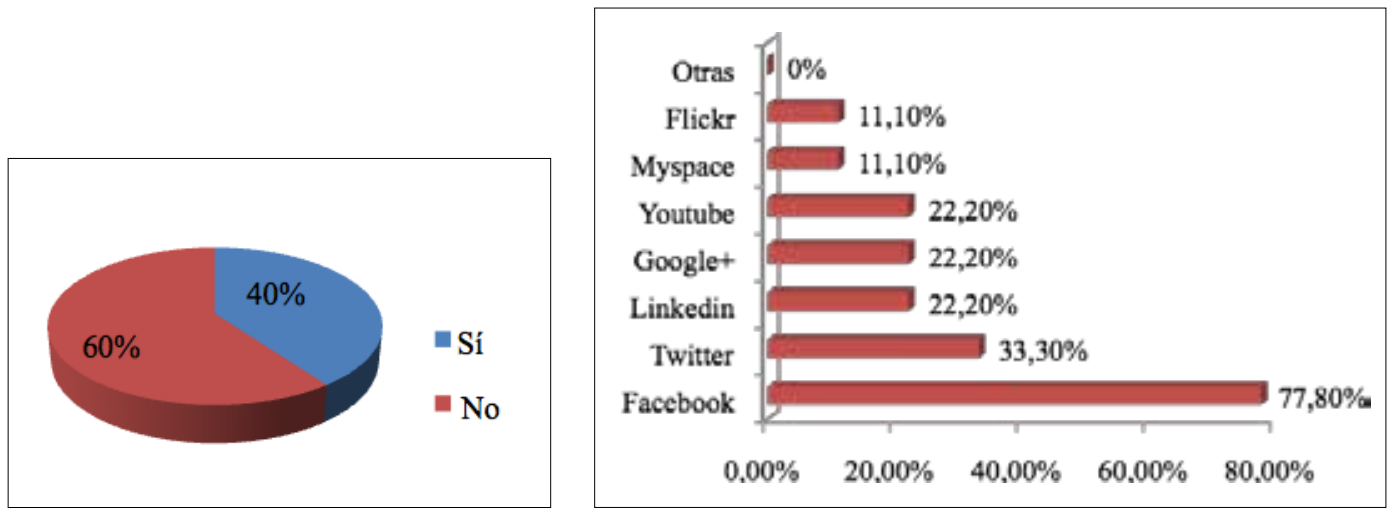

Fuente: Elaboración propia

Los motivos de uso más frecuentes de estas aplicaciones de la Web 2.0, han sido mayoritariamente como métodos de promoción y publicidad: para informar sobre sus productos y servicios turísticos $(77,8 \%)$, para ofrecer promociones y descuentos $(77,8 \%)$ y para intentar llegar a un mayor número de personas $(77,8 \%)$. 
Por el contrario podemos observar que apenas se utilizan como canal de comercialización para personalizar el servicio a través de la red $(11,1 \%)$ o para controlar la confianza y la imagen de marca de la agencia $(22,2 \%)$. Es por ello, por lo que están perdiendo una gran oportunidad de generar una reputación on-line y el reconocimiento de su marca

Gráfico 4: ¿Para qué utilizan estas agencias las Redes Sociales?

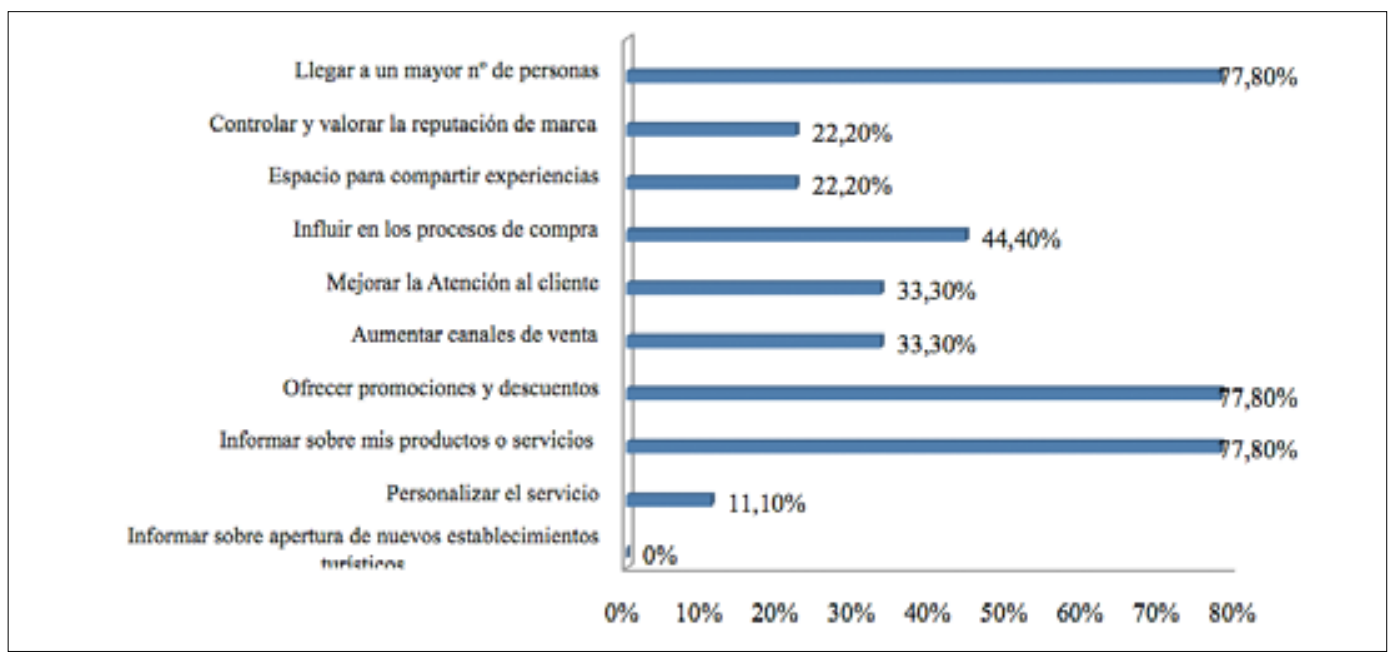

Fuente: Elaboración propia

En cuanto a los métodos de cara a la promoción y captación de clientes, el más utilizado son las campañas de publicidad en medios de comunicación, escogido en un $71,4 \%$ de los casos. Esto se debe a que la mayoría de las agencias locales de la ciudad dependen de la promoción que hace el grupo corporativo al que pertenecen o la sede central de su marca. Le sigue la promoción en la página web propia de la agencia (61,9\%), los e-mails $(57,1 \%)$ y los mailings directos /personalizables $(52,4 \%)$ y los conocidos catálogos, ambos en un $52,4 \%$ de los casos. Las Redes Sociales sólo se utilizan en un 28,57\%. Otras formas de promoción $(19,05 \%)$ para algunas agencias son el sms, revistas propias y la transmisión "boca a boca".

Gráfico 5: Métodos de comercialización que utilizan

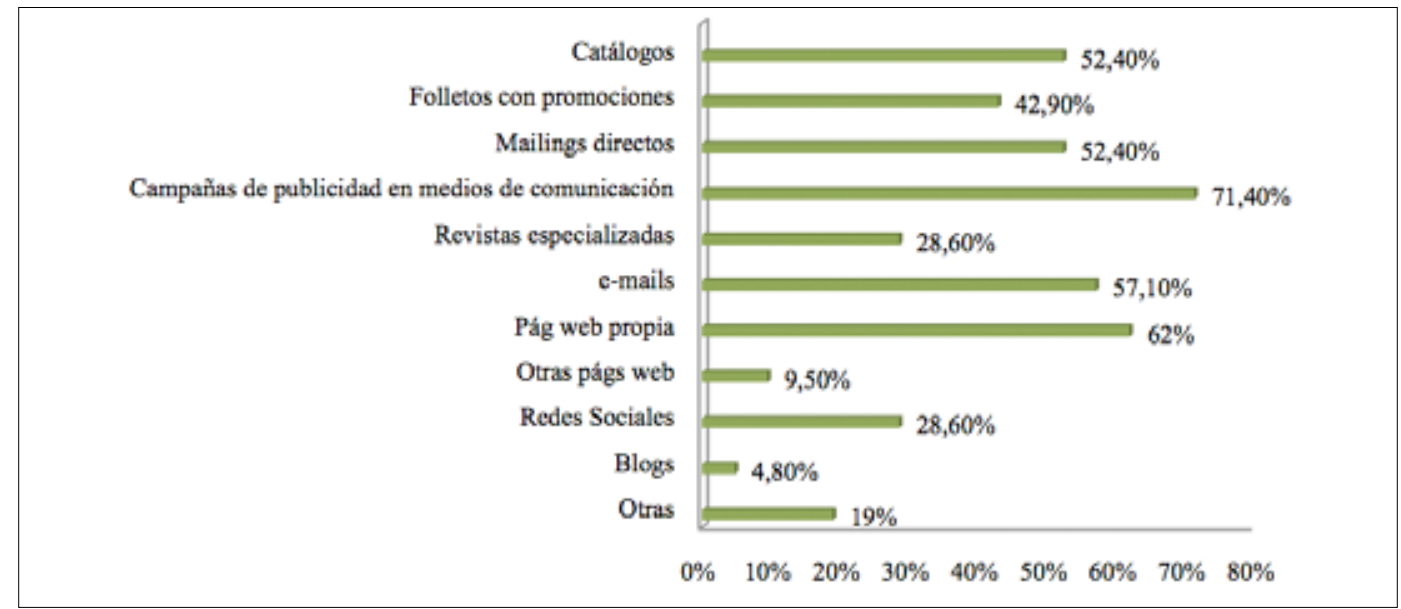

Fuente: Elaboración propia 
Finalmente se ha pedido a todas las agencias participantes en la encuesta, que den su opinión acerca de cuál piensan que es el futuro de las Agencias de Viajes tradicionales tal y como las conocemos. El $60 \%$ opina que tienden a un cambio en el modelo de negocio, mientras que un $40 \%$ que cree que tienden a centrarse en nichos de mercado muy específicos. Además ningún encuestado cree que lleguen a ser exclusivamente virtuales y muy pocos opinan que vaya a producirse ningún cambio en su modelo de negocio (5\%) o que vayan a desaparecer tal y como las conocemos ahora (15\%).

\section{Conclusiones e implicaciones}

Esta investigación ha tratado de relacionar los conceptos del turismo, las Agencias de Viajes tradicionales y las Redes Sociales.

Tras el estudio queda claro que las nuevas tecnologías han cambiado completamente el mercado de los viajes. Los negocios turísticos han experimentado cambios en las formas de hacer publicidad, de comercializar los productos y servicios turísticos, de influir en los consumidores y de obtener un feedback de las experiencias de los usuarios. Los clientes están mucho más informados y son más exigentes a la hora de tomar sus decisiones de compra, es por ello que son mucho más activos gracias a las Redes Sociales. Las agencias de viajes tienen que aprovechar esta oportunidad de "entrar en el juego" para posicionarse interactuando con los clientes, además de beneficiarse de la reducción de costes que supone y del acceso a un enorme mercado de usuarios potenciales.

Con los resultados obtenidos en esta investigación, se ve la clara relación que debe de existir entre las Agencias de Viajes y las Redes Sociales, sin embargo las agencias de la ciudad de Ourense todavía no han asumido este nuevo rol que las TIC les proporcionan.

El 60\% de las agencias encuestadas no está presente en las Redes Sociales, y si no se adaptan a la Web 2.0 no sólo van a perder una cuota de mercado importante, sino que ponen en riesgo su supervivencia. Necesitan un cambio en el modelo de negocio tradicional y deben proporcionar unos servicios diferenciadores frente a otras agencias. La clave es combinar la presencia en Internet y en las Redes Sociales con una atención personalizada en la oficina a pie de calle. El estar presente on-line les va a permitir comunicarse con los clientes, fidelizarlos, marcar la diferencia y especializarse, además de conseguir una buena reputación e imagen de marca innovadora en la red.

La mejor manera de hacerlo es maximizando el uso de las Redes Sociales como herramientas eficaces de marketing y aprovechar el feedback que proporcionan para mejorar en las nuevas campañas. En resumen hay que destacar que la clave está básicamente en conversar y en cuidar a los clientes. La agencia debe buscar su visibilidad en la red, creando contenido interesante y generando una "historia" alrededor de la marca que le permita conseguir una interacción constante con los clientes, lo que va a incidir de manera directa en su reputación on-line.

Por otro lado, existe una tendencia generalizada a minusvalorar el beneficio económico que se obtiene estando en las Redes Sociales. La mayoría no puede cuantificar este dato y no tiene claro que los beneficios sean inmediatos, sin embargo el simple ahorro en publicidad e imagen de marca resulta evidente. También se han detectado ciertos inconvenientes con las agencias de grandes marcas nacionales tipo El Corte Inglés, cuyas webs o Redes Sociales son del grupo al que pertenecen, lo que impide un trato personalizado a través de la "sucursal". Además los directivos de la mayoría de ellas suelen ser ya personas con años de experiencia y edad avanzada que han vivido el negocio desde un punto de vista tradicional y que no están muy convencidos del futuro y el éxito del negocio con ayuda de las Redes Sociales. No obstante, la mayoría de los grupos minoristas más conocidos en España como Muchoviaje, Vibo Viajes, eDreams, Halcón Viajes, Rumbo, etc. ya han apostado por las Redes Sociales como un elemento fundamental para relacionarse con sus clientes.

El cliente que acude a la Agencia de Viajes tradicional suele ser un cliente que todavía desconfía de Internet y de los sistemas de pago electrónicos, pero con la revolución que actualmente estamos viviendo, cada vez más gente utiliza la red para operaciones de compra/venta y para acceder a los servicios que el sector turístico proporciona on-line. Así que poco a poco los usuarios de Internet se están familiarizando con el entorno y van ganando confianza con los sistemas de comercio electrónico, lo que acabará redundando en su uso masivo.

Si no se produce un cambio en el sector, parece que la agencias, tal y como se conocen, están destinadas a la desaparición a corto o a medio plazo, pese a que algunas crean firmemente que el modelo de negocio conocido de Agencia de Viajes tradicional, va a seguir funcionando con el paso de los años, 
pese a la revolución de las TIC y a la crisis económica. Al no asumir el cambio otras agencias tomarán el relevo, puesto que ya están marcando la diferencia a través de las Redes Sociales.

A pesar de que estén muy bien valoradas por los clientes, no deberían de aferrarse solamente al índice de satisfacción. Su futuro pasa porque busquen posicionarse allí donde están los posibles clientes potenciales, y la mejor manera es a través de las Redes Sociales. Es una nueva forma de relacionarse con el cliente.

A modo de conclusión final hay que destacar que las Agencias de Viajes tradicionales tienen que apostar fuertemente por los nuevos medios y tecnologías de distribución on-line, sacándoles el máximo partido posible y publicitándose con mejoras que resalten la contratación individualizada. Ofreciendo su experiencia y con la garantía de ser eficientes en su trabajo, serán capaces de prestar un mejor servicio $\mathrm{y}$, consecuentemente, de generar un mayor valor añadido para el consumidor final.

\section{Bibliografia}

Anderson, Paul

2007. "What is Web 2.0? Ideas, technologies and implications for education". JISC Technologies and

Standards Watch.

Aruguete, G.

2001. Redes sociales. Una propuesta organizacional alternativa.

Boyd, D.; Ellison, N.

2008. "Social Network Sites: Definition, History and Scholarship". Journal of Computer-ediated communication, 13: 210-230.

Chung, Y. J.; Buhalis, D.

2008. "Information needs in online social networks". Information Technology \& Tourism, 10, (4): 267-281.

De Vicente, J.L.

2005. La Inteligencia Colectiva en la Web 2.0.Creación e Inteligencia Colectiva, catálogo del Séptimo

Festival Zemos 98: 95-100

Granovetter, Mark

1973. The Strength of Weak Ties. American Journal of Sociology 78 (6): 1360-1380.

Jeacle, i.; Carter, c.

2011. "In TripAdvisor we trust: Rankings, calculative regimes and abstract system. Accounting,

Organizations and Society 36: 293-309.

Mildgram, Stanley.

1967. "The Small-World Problem". Psychology Today, 1(1): 60-67.

O'reilly, Tim.

2006. What Is Web 2.0? Design Patterns and Business Models for the Next Generation of Software.

O'Reilly Media Network.

Súarez y Alonso, Ramón c.

2007. Tecnologías de la Información y la Comunicación: Introducción a los Sistemas de Información y de Telecomunicación. Vigo: Ideaspropias Editorial.

VVogeler Ruiz, c.; Hernández Armand, E.

2000. El Mercado Turístico: Estructura, Operaciones y Procesos de Producción (1 ${ }^{\mathrm{a}}$ ed.). Madrid: Editorial

Centro de Estudios Ramón Areces

\section{Recursos electrónicos}

Google Académico: http://scholar.google.es

Hosteltur: http://www.hosteltur.com

El Blog de Jimmy Pons: http://jimmypons.typepad.com/

El Blog de Tirso Maldonado: http://tirsomaldonado.wordpress.com/

O'Reilly Media: http://oreilly.com

Diseño de encuestas: http://e-encuesta.es 